\title{
Bioconjugate structures vs. composite nanoparticulate carriers: the battle for the future of smart, effective and safe cancer management
}

\author{
Mohamed Ismail Nounou \\ Department of Pharmaceutical Sciences, School of Pharmacy, University of Saint Joseph, Hartford, CT 06103, USA.
}

Correspondence to: Dr. Mohamed Ismail Nounou, Department of Pharmaceutical Sciences, School of Pharmacy, University of Saint Joseph, Hartford, CT 06103, USA. E-mail: nounou@usj.edu

\begin{abstract}
How to cite this article: Nounou MI. Bioconjugate structures vs. composite nanoparticulate carriers: the battle for the future of smart, effective and safe cancer management. J Cancer Metastasis Treat 2018;4:29.

http://dx.doi.org/10.20517/2394-4722.2018.18
\end{abstract}

Received: 2 Feb 2018 First Decision: 23 Apr 2018 Revised: 25 Apr 2018 Accepted: 6 Jun 2018 Published: 21 Jun 2018

Science Editor: Lucio Miele Copy Editor: Jun-Yao Li Production Editor: Cai-Hong Wang

For the past couple of decades, academic research has been mainly focusing on novel carrier systems and nanoparticulate colloidal technologies for drug delivery, such as nanoparticles, nanospheres, vesicular systems, liposomes, nanocapsules, etc. Such efforts aided in the creation of newly marketed products such as Doxil $^{\circledR}$ in the market ${ }^{[1,2]}$. Such systems provide the tools to customize a superior drug delivery system, impart novel functions to old drugs such as longer half-life and stealth properties (as in the case of Doxil ${ }^{\circ}$ ), and provide them with either passive or active targeting properties via grafting the carrier system with targeting moieties and/or imaging agents or another drug within the same carrier system ${ }^{[3]}$. Such technologies opened the gate towards more sophisticated and effective multi-acting platform(s) which can offer site-targeting, imaging, and treatment using a single multi-functional system ${ }^{[4]}$. Unfortunately, such technologies are faced with major problems including low stability profile, short shelf-life, and poor reproducibility across and within production batches leading to harsh bench-to-bedside transformation. The commercial scale-up processes of composite nanoparticulate carriers are challenging, time-consuming and costly. Such scale-up processes from the bench to pilot small-scale production, and subsequently to the full-scale process involve significant major pre-formulation and formulation developmental steps along with the design of rugged and robust in vitro characterization techniques to ensure safety and efficacy of the final formulation along with quantitative determination of intra/inter-batch variability to comply with pharmacopeial standards and regulations. Additionally, the majority of such novel therapeutic systems' inactive adjuvants and reagents used in the pre-formulation and formulation steps are not yet approved by the FDA and not listed in their approved inactive ingredient database (IID).

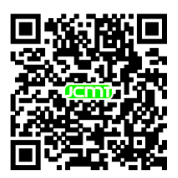




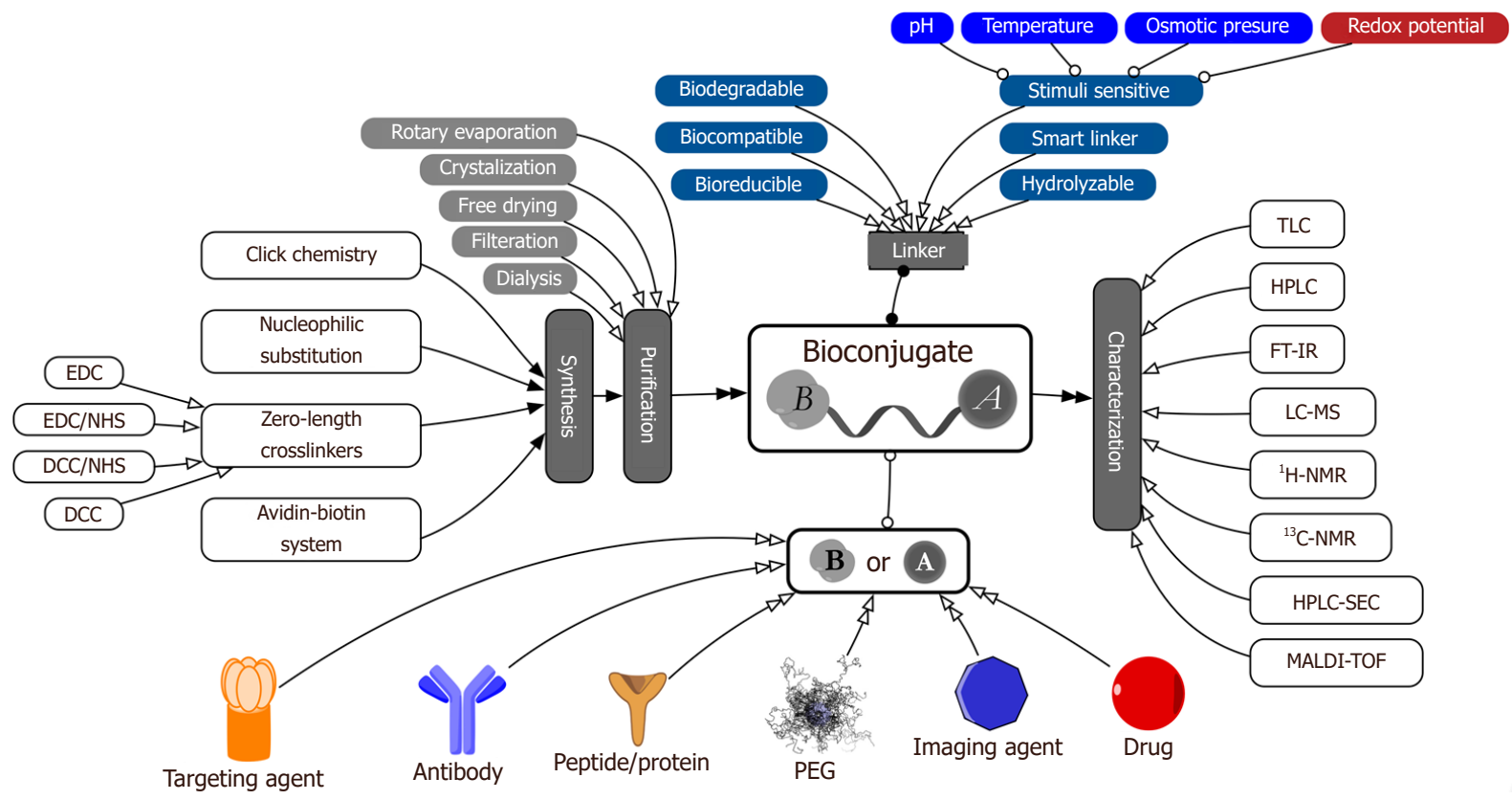

Figure 1. Schematic diagram of bioconjugates' structure, design, synthesis, purification and characterization

Initiatives to overcome such setbacks either involved the design and development of novel new chemotherapeutic agents, chemical or physical modifications of currently used chemotherapeutic agents or novel smart bioconjugates ${ }^{[5]}$. Currently, pharmaceutical industry along with academic research is investing heavily in bioconjugate structures. The major purpose of bioconjugation is to create a stable conjugate between two molecules via a covalent link, at least one of which is a biomolecule ${ }^{[6]}$. By design, the covalent linkage should be easily biologically-cleavable to enable the release of the bioactive molecule at the desired target site. The main advantages of bioconjugation and the generated biomolecules include enhanced physical and chemical stability in the active pharmaceutical ingredient (API) journey to the target site, providing better safety and efficacy profiles, delivering enhanced API protection against proteolysis and immune responses and enhancement of the targeting powers of such novel bioconjugates nanoparticulate systems ${ }^{[6]}$.

Bioconjugate technologies offer an appealing and advantageous alternative to nanoparticulate delivery systems with all its flexible benefits when it comes to customized design and tailored grafting along with avoiding most of its shortcomings. Bioconjugates offer the flexibility in customized designing of personalized products. Bioconjugates facilitate simple and easy drug (active pharmaceutical ingredient) conjugation, using various smart biocompatible, bioreducible, or biodegradable linkers, to targeting agents, PEG layer or another drug [Figure 1]. Such technology enables the formation of smart multi-functional platform(s) offered by nanoparticulate carriers and bioconjugates structures. Furthermore, conjugates are still considered chemical compounds. This fact simply allows the use of traditional analytical and manufacturing technologies in the characterization and manufacturing of traditional active pharmaceutical ingredients offering high probability for their successful transition from bench to bedside. Moreover, the final formulation could be a simple injectable or solid formulation, which offers long shelf-life and enhanced stability profile.

Subsequently, bioconjugation technologies can aid in creating safer, cheaper, stable, and effective novel therapeutics. It can also be a rate-limiting step in reinventing old drugs and imparting new functions to them that would enhance their targetability, pharmacokinetic and pharmacodynamic parameters, and their overall formulation patient compliance, easing their transition to market ${ }^{[7]}$. A major focus should be the transformation of such novel bioconjugates' technologies from bench to bedside. The use of click chemistry, bioconjugation technologies, ligand post-insertion and labeling techniques need to be extensively 
researched for ease of scale-up and proper bench-to-bedside transformation. Consequently, a current focus is on simple bioconjugate structures, which can be easily synthesized with high yield, reduced cost and high stability profile of the final formulation. This could provide a practical direction for the development of novel management tools and therapeutics, paving the road to affordable, scalable, stable, efficient and safe diseasemanagement strategies.

\section{DECLARATIONS}

\section{Authors' contributions}

Nounou MI conceived the presented idea, developed the theoretical formalism and fully contributed to the writing of the manuscript.

\section{Availability of data and materials}

Not applicable.

\section{Financial support and sponsorship}

None.

\section{Conflicts of interest}

The author declares that there are no conflicts of interest.

\section{Ethical approval and consent to participate}

Not applicable.

\section{Consent for publication}

Not applicable.

\section{Copyright}

(c) The Author(s) 2018.

\section{REFERENCES}

1. Muggia FM. Doxil in breast cancer. J Clin Oncol 1998;16:811-2.

2. Porche DJ. Liposomal doxorubicin (doxil). J Assoc Nurses AIDS Care 1996;7:55-9.

3. Tagami T, Ozeki T. Recent trends in clinical trials related to carrier-based drugs. J Pharm Sci 2017;106:2219-26.

4. Cagel M, Tesan FC, Bernabeu E, Salgueiro MJ, Zubillaga MB, Moretton MA, Chiappetta DA. Polymeric mixed micelles as nanomedicines: achievements and perspectives. Eur J Pharm Biopharm 2017;113:211-28.

5. Lammers T, Kiessling F, Hennink WE, Storm G. Drug targeting to tumors: principles, pitfalls and (pre-) clinical progress. J Control Release 2012;161:175-87.

6. Hermanson GT. Bioconjugate Techniques, 3rd Edition. Academic Press; 2013.

7. Duncan R. Polymer conjugates as anticancer nanomedicines. Nat Rev Cancer 2006;6:688-701. 